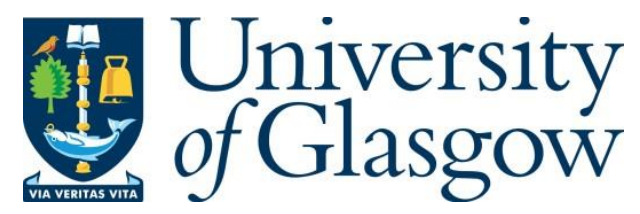

Yang, X., Fang, M., Ren, A., Zhang, Z., Abbasi, Q. H., Alomainy, A., Mehran, K. and Hao, Y. (2017) Reverse recognition of body postures using on-body radio channel characteristics. IET Microwaves, Antennas and Propagation, 11(9), pp. 1212-1217.

This paper is a postprint of a paper submitted to and accepted for publication in IET Microwaves, Antennas and Propagation and is subject to Institution of Engineering and Technology Copyright. The copy of record is available at the IET Digital Library

There may be differences between this version and the published version. You are advised to consult the publisher's version if you wish to cite from it.

http://eprints.gla.ac.uk/141103/

Deposited on: 21 July 2017

Enlighten - Research publications by members of the University of Glasgow http://eprints.gla.ac.uk 


\title{
Reverse Recognition of Body Postures Using On-body Radio Channel Characteristics
}

\author{
Xiaodong Yang ${ }^{1 *}$, Meiling Fang ${ }^{1}$, Aifeng Ren ${ }^{1}$, Zhiya Zhang ${ }^{1}$, Qammer Hussain Abbasi $^{2}$, \\ Akram Alomainy ${ }^{3}$, Kamyar Mehran ${ }^{3}$, and Yang $\mathrm{Hao}^{3}$ \\ ${ }^{1}$ School of Electronic Engineering, Xidian University, Xi'an 710071, China. \\ ${ }^{2}$ Department of Electrical and Computer Engineering, Texas A\&M University at Qatar, Doha \\ 22880, Qatar. \\ ${ }^{3}$ School of Electronic Engineering and Computer Science, Queen Mary, University of \\ London, London E1 4NS, UK. \\ *Corresponding author: xdyang@xidian.edu.cn
}

\begin{abstract}
AL). In most cases, radio channel models for WBANs are fixed when a specific body posture is considered. To the best of our knowledge, little work has been done on the reverse body posture information extraction using WBAN radio channel characteristics. This paper aims to classify human postures from on-body narrowband wireless channel information. It is demonstrated that by applying the random forest (RF) classification technique, the action of the human body can be detected. The classification error is perfectly acceptable for RF algorithm. Two propagation environments were compared and the results indicate that the classification error is less in the anechoic chamber (21.39\%). In summary, this paper provides a novel approach to detect human body postures by using body-centric wireless channel information, and will be beneficial for AL.
\end{abstract}

Keywords: assisted living, on-body narrowband wireless channels, posture detection, random forest

\section{Introduction}

\subsection{Motivation}

Miniaturization of electronic devices and advanced research and development in body-worn hardware, embedded software, digital signal processing and biomedical engineering have made the concept of bodycentric wireless communication (BCWC) practically possible [1]. BCWC has a wide range of potential application such as in smart home, healthcare and so forth. In recent years, assisted living (AL) is gaining more and more interest, both in academia and industry. AL is very useful in preventing, curing, and improving the wellness and health conditions of older adults [2]. Daily activity monitoring, which can be used for detecting non-normal behaviour of older adults, is the key issue in AL technology. Posture detection, which is the key point in monitoring of activities, plays an important role in AL. By collecting on-body channel data (or received signal strength), a home care robot can response in time and take 
necessary actions; on the other hand, the data collected by a potential smart home will be transmitted to a corresponding doctor to make a timely diagnosis (Figure 1).

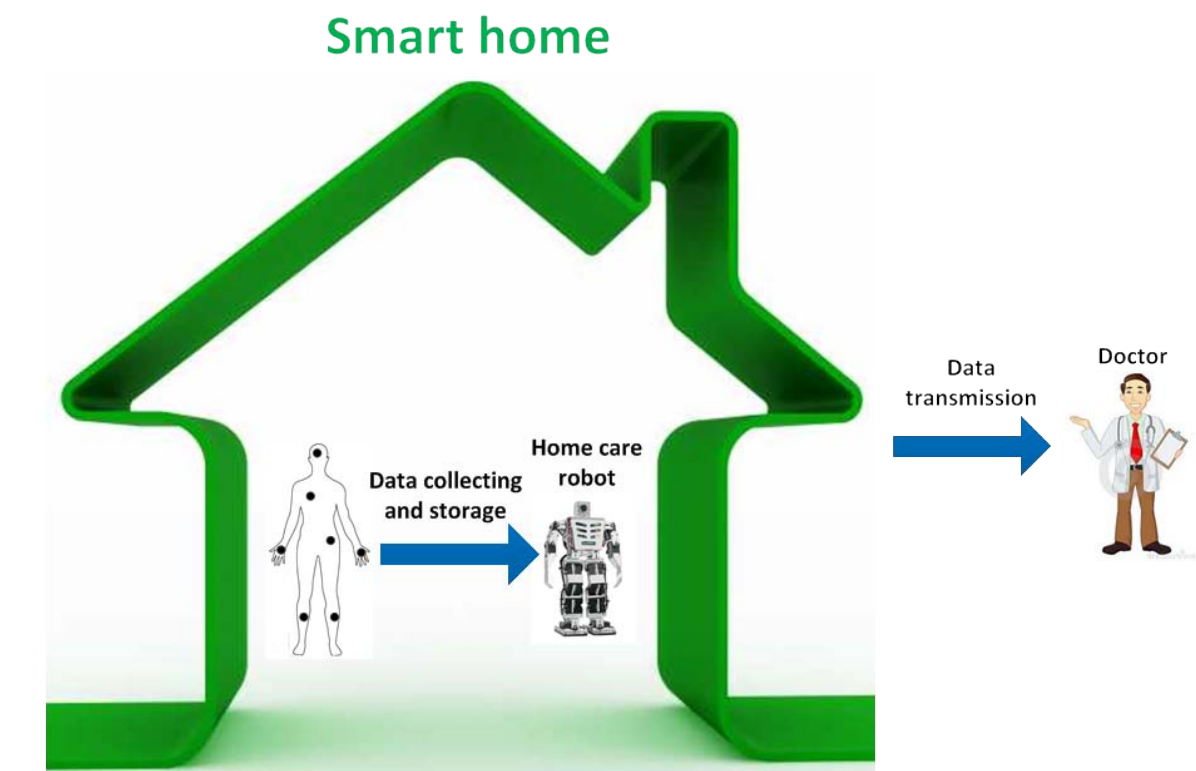

Figure 1 Posture detection using on-body narrowband wireless channels in assisted living

In this paper, we aimed at classifying postures from on-body narrowband channel parameters. The posture of the subject can be detected from the channel-path gain information of on-body nodes. The paper is organized as follows. The rest of this section introduces some related work about on-body narrowband wireless communications and posture recognition. Section 2 gives the basics of decision-tree combination. Section 3 realizes activity classification by using random forest. In section 4, conclusions are given.

\subsection{Related work}

Human-posture detection is now widely explored in some literatures. In this section, these works are reviewed. Then the necessity and importance of the work are discussed. Computer-vision approach, wireless sensor network techniques, and other advanced techniques are now widely used in human-posture detection. Tahir et al. [3] developed a framework to efficiently match the human postures. In [4], the authors proposed a novel method to detect various posture-based events in a typical elderly monitoring application in a home surveillance scenario. Akmeliawati et al. [5] introduce the gesture and hand posture tracking systems for a prototype real-time New Zealand sign language recognition system. In [6], a finite state and fuzzy logic based approach to hand gesture learning and recognition is proposed. Bilal et al. [7] introduced an algorithm that is capable to recognize hand posture in a sophisticated background. In [8], the traditional worm's detection approaches is introduced. In [9], Bilal et al. show that extracting features from hand shape is so essential during recognition stage for applications such as SL translators. In [10], 
the development of an abnormal posture detection device intended for home use is described. Gjoreski et al. [11] present an approach to fall detection with accelerometers that exploits posture recognition to identify postures that may be the result of a fall. In [12], the authors propose a method to automatically detect human poses in a single image, based on a 2D model combined with anthropometric data. Marek et al. [13] describe the approach used to detect hand postures in computationally undemanding manner. In [14], a model to reconstruct 3D virtual humans based on a single and spontaneous image is presented. Putchana et al. [15] proposed a simple wireless intelligent system prototype for fall detection and movement classification for real-time monitoring of the elderly. In [16], the authors have designed and implemented a wireless EMG, which can acquire the signal from six muscles in the back. Alvaro et al. [17] implemented a tool for detecting posture variation of seated person in front of a computer with the goal of monitoring when it deviated from its correct position. In [18], the authors present a novel appearance based method to recognize and track human hand in an unknown environment without any constraints on hand in an unknown environment without any additional devices. Shiravandi et al. [19] present a method for hand gesture recognition using dynamic Bayesian networks. In [20], a prototype system of fall detection application designed for smart phone is proposed. Sawasdee et al. [21] propose a hand posture recognition as an assistive tool for elderly care. In [22], a drowsy driver detection system has been developed, using video processing analyzing eyes blinking concepts for measuring eyes closure duration and head posture estimation to verify the driver vigilance state. In [23],a motion capture system was developed using the Microsoft Kinect depth camera, results are included for different hand postures of participating pianists.

The related works presented above provide us with important inspiration regarding human-posture detection. It is found that there are few works achieving posture recognition using body-centric wireless channel information which can be obtained through frequency domain channel measurement. As a new mechanism of detecting human-posture changes, the approach and train of thought presented in this paper can be seen as an important supplement of the available ways of achieving human-posture detection.

\section{Basics of random forest (RF) framework}

Decision tree is a method of dividing the space using hyper plane; for each segmentation, the current space is divided into two parts. Therefore, each leaf node is located in a disjoint region in the space; in the process of decision-making, the feature value for each dimension of the input samples will be considered. Finally, the sample would fall into one of the $\mathrm{N}$ regions (It is assumed that there are $\mathrm{N}$ leaf nodes). The random forests (RF), first proposed by Leo Breiman [24], are composed of many decision trees; each 
decision tree is independent from the others. To construct random forests [24], firstly, samples are selected from the original training set using bootstrap; then, decision tree models should be established to match the number of samples; finally, the ultimate classification results can be decided according to the voting results of the previous classification. In the process of establishing each decision tree, sampling and complete segmentation should be noticed. For row sampling, there might be repeated samples in the obtained sample set. We assume that there are $\mathrm{N}$ input samples, then, the number of samples for sampling is $\mathrm{N}$. Thus, the input samples for each tree are not all the samples, avoiding the over-fitting. In the column sampling, features should be selected; it should be noted that the number of selected features should be much less than that of total features. Then the decision tree can be established in the way of complete segmentation for the data after sampling; thus, either a leaf node in the decision tree is indivisible or all the samples in it belong to the same classification. RF is a data-driven non-parametric approach; it does not need any prior knowledge.

The basic steps to construct an RF are summarized as follows:

Step 1: Selected samples, which are also the samples at the root nodes of decision tree, are used to train a decision tree.

Step 2: If each sample has $L$ properties, in the process of split, $l(l<<L)$ properties are selected from $L$ properties randomly. Then a certain strategy is employed to decide the split property for the corresponding node.

Step 3: Every node in a decision tree should split following the approach presented in step 2; it should be noted that pruning does not take place in the whole process of decision-tree forming. Finally, random forest is formed by constructing a large number of decision trees.

In this work, the number of trees is defined as 1000; and $l$ properties, is defined as 3 ( $l$ is usually taken as the root mean square of $L$ ). The framework of random forest-based posture detection is given below (Figure 2). 


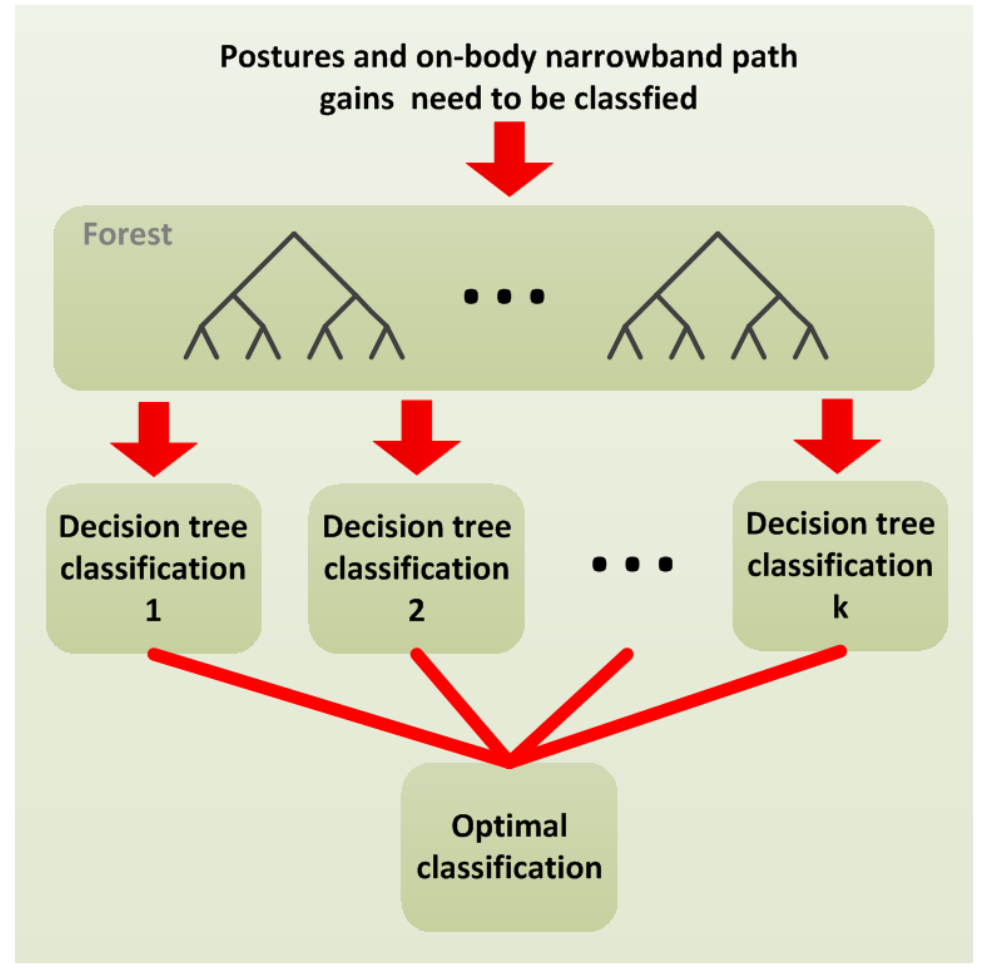

Figure 2 Random-forest framework for posture detection considering on-body narrowband wireless channels

Table 1(a) Confusion matrix for the anechoic chamber environment (number of trees=1000, number of variables tried at each split=2).

The OOB estimate of error rate is $21.39 \%$.

\begin{tabular}{llllllllllllllllllll}
\hline & $\mathrm{A}$ & $\mathrm{B}$ & $\mathrm{C}$ & $\mathrm{D}$ & $\mathrm{E}$ & $\mathrm{F}$ & $\mathrm{G}$ & $\mathrm{H}$ & $\mathrm{I}$ & $\mathrm{J}$ & $\mathrm{K}$ & $\mathrm{L}$ & $\mathrm{M}$ & $\mathrm{N}$ & $\mathrm{O}$ & $\mathrm{P}$ & $\mathrm{Q}$ & $\mathrm{R}$ \\
$\mathrm{A}$ & 20 & 0 & 0 & 0 & 0 & 0 & 0 & 0 & 0 & 0 & 0 & 0 & 0 & 0 & 0 & 0 & 0 & 0 & \\
$\mathrm{~B}$ & 3 & 16 & 1 & 0 & 0 & 0 & 0 & 0 & 0 & 0 & 0 & 0 & 0 & 0 & 0 & 0 & 0 & 0 & \\
$\mathrm{C}$ & 0 & 2 & 17 & 0 & 0 & 0 & 0 & 0 & 0 & 0 & 0 & 0 & 0 & 0 & 1 & 0 & 0 & 0 & \\
$\mathrm{D}$ & 0 & 0 & 1 & 19 & 0 & 0 & 0 & 0 & 0 & 0 & 0 & 0 & 0 & 0 & 0 & 0 & 0 & 0 & \\
$\mathrm{E}$ & 0 & 0 & 0 & 2 & 17 & 0 & 1 & 0 & 0 & 0 & 0 & 0 & 0 & 0 & 0 & 0 & 0 & 0 & \\
$\mathrm{~F}$ & 0 & 0 & 0 & 0 & 1 & 19 & 0 & 0 & 0 & 0 & 0 & 0 & 0 & 0 & 0 & 0 & 0 & 0 \\
$\mathrm{G}$ & 0 & 0 & 0 & 0 & 0 & 1 & 18 & 1 & 0 & 0 & 0 & 0 & 0 & 0 & 0 & 0 & 0 & 0 & \\
$\mathrm{H}$ & 0 & 0 & 0 & 0 & 0 & 0 & 2 & 17 & 1 & 0 & 0 & 0 & 0 & 0 & 0 & 0 & 0 & 0 \\
$\mathrm{I}$ & 0 & 0 & 0 & 0 & 0 & 0 & 0 & 2 & 18 & 0 & 0 & 0 & 0 & 0 & 0 & 0 & 0 & 0 \\
$\mathrm{~J}$ & 0 & 0 & 0 & 0 & 0 & 0 & 0 & 0 & 1 & 19 & 0 & 0 & 0 & 0 & 0 & 0 & 0 & 0 \\
$\mathrm{~K}$ & 0 & 0 & 0 & 0 & 0 & 0 & 0 & 0 & 0 & 2 & 17 & 1 & 0 & 0 & 0 & 0 & 0 & 0 \\
$\mathrm{~L}$ & 0 & 1 & 1 & 0 & 0 & 0 & 0 & 1 & 0 & 2 & 3 & 7 & 0 & 1 & 0 & 1 & 0 & 3 \\
$\mathrm{M}$ & 0 & 0 & 1 & 1 & 0 & 0 & 0 & 0 & 1 & 0 & 1 & 0 & 14 & 1 & 0 & 0 & 0 & 1 \\
$\mathrm{~N}$ & 0 & 0 & 0 & 0 & 0 & 0 & 0 & 0 & 0 & 0 & 0 & 0 & 2 & 17 & 1 & 0 & 0 & 0 \\
$\mathrm{R}$ & 0 & 0 & 0 & 1 & 1 & 0 & 0 & 0 & 0 & 0 & 0 & 0 & 0 & 0 & 3 & 13 & 0 & 0 & 2
\end{tabular}


Table 1(b) Confusion matrix for the indoor environment (number of trees=1000, number of variables tried at each split=2). The OOB estimate of error rate is $25.83 \%$.

\begin{tabular}{|c|c|c|c|c|c|c|c|c|c|c|c|c|c|c|c|c|c|c|}
\hline & A & B & $\mathrm{C}$ & D & $E$ & $F$ & G & $\mathrm{H}$ & I & $\mathrm{J}$ & $\mathrm{K}$ & L & $\mathrm{M}$ & $N$ & 0 & $\mathrm{P}$ & $Q$ & $\mathrm{R}$ \\
\hline A & 20 & 0 & 0 & 0 & 0 & 0 & 0 & 0 & 0 & 0 & 0 & 0 & 0 & 0 & 0 & 0 & 0 & 0 \\
\hline B & 1 & 17 & 0 & 0 & 0 & 0 & 0 & 0 & 0 & 0 & 0 & 0 & 0 & 0 & 0 & 1 & 1 & 0 \\
\hline C & 0 & 1 & 17 & 0 & 1 & 0 & 0 & 0 & 0 & 0 & 0 & 1 & 0 & 0 & 0 & 0 & 0 & 0 \\
\hline D & 0 & 0 & 2 & 16 & 0 & 0 & 0 & 0 & 0 & 0 & 0 & 0 & 1 & 0 & 0 & 0 & 1 & 0 \\
\hline$E$ & 0 & 0 & 0 & 2 & 15 & 0 & 0 & 2 & 0 & 0 & 0 & 0 & 0 & 0 & 0 & 0 & 1 & 0 \\
\hline $\mathrm{F}$ & 0 & 0 & 0 & 0 & 2 & 16 & 0 & 0 & 0 & 0 & 0 & 2 & 0 & 0 & 0 & 0 & 0 & 0 \\
\hline $\mathrm{G}$ & 0 & 0 & 0 & 0 & 0 & 1 & 19 & 0 & 0 & 0 & 0 & 0 & 0 & 0 & 0 & 0 & 0 & 0 \\
\hline $\mathrm{H}$ & 0 & 0 & 0 & 0 & 0 & 0 & 1 & 18 & 0 & 0 & 0 & 0 & 0 & 0 & 1 & 0 & 0 & 0 \\
\hline I & 0 & 0 & 0 & 0 & 0 & 1 & 0 & 2 & 17 & 0 & 0 & 0 & 0 & 0 & 0 & 0 & 0 & 0 \\
\hline $\mathrm{J}$ & 0 & 0 & 0 & 0 & 0 & 0 & 0 & 1 & 1 & 16 & 0 & 0 & 1 & 0 & 1 & 0 & 0 & 0 \\
\hline $\mathrm{K}$ & 0 & 0 & 0 & 0 & 0 & 0 & 0 & 0 & 0 & 1 & 18 & 1 & 0 & 1 & 0 & 0 & 0 & 0 \\
\hline L & 0 & 1 & 0 & 0 & 0 & 4 & 0 & 2 & 0 & 0 & 2 & 9 & 0 & 0 & 1 & 0 & 0 & 1 \\
\hline $\mathrm{M}$ & 0 & 0 & 0 & 1 & 0 & 0 & 0 & 1 & 0 & 0 & 0 & 0 & 12 & 0 & 4 & 1 & 0 & 1 \\
\hline $\mathrm{N}$ & 0 & 0 & 0 & 0 & 0 & 0 & 0 & 0 & 0 & 2 & 0 & 0 & 2 & 16 & 0 & 0 & 0 & 0 \\
\hline 0 & 0 & 0 & 0 & 1 & 0 & 0 & 0 & 0 & 0 & 1 & 0 & 1 & 3 & 3 & 9 & 0 & 0 & 2 \\
\hline $\mathrm{P}$ & 0 & 1 & 0 & 0 & 0 & 0 & 0 & 0 & 0 & 0 & 0 & 0 & 1 & 0 & 2 & 15 & 1 & 0 \\
\hline$Q$ & 1 & 3 & 1 & 1 & 0 & 0 & 0 & 0 & 0 & 0 & 0 & 2 & 2 & 0 & 0 & 3 & 7 & 0 \\
\hline $\mathrm{R}$ & 0 & 1 & 0 & 0 & 0 & 0 & 0 & 0 & 1 & 0 & 0 & 1 & 1 & 0 & 1 & 1 & 4 & $\begin{array}{l}1 \\
0\end{array}$ \\
\hline
\end{tabular}

\section{Posture detection using random forest}

\subsection{Out-of-bag (OOB) error}

Random forest is the combination of decision trees. The training set is generated by using a bagging approach; the probability that each sample in the original training set will not be selected is $(1-1 / N)^{N}$, where $N$ is the number of samples. This indicates that almost $1 / 3$ of the samples in the original sample set will not be in the bootstrap sample set. These data are known as out-of-bag (OOB) data and can be used to evaluate the performance of the model. For each decision tree, an OOB error estimation can be obtained. The generalization error estimation of the random forest can be obtained by taking the average of all OOB error estimations. It has been demonstrated $[25,26]$ that OOB estimation can be considered as the method for generalization error estimation. Breiman [27] investigated the error estimation for bagged classifier, and demonstrated that the precision for OOB estimation is the same with the test set. Therefore, in this work, OOB error estimation is used as the primary method for model performance assessment.

The steps to obtain OOB estimation can be summarized as follows: 
Step 1: For the given RF, use out-of-bag data to test its performance. Assume that the number of OOB data is $S$; these OOB data are the input. Substitute these data into the RF classifier; the classifier would give the corresponding classification.

Step 2: Because the type of data is known, compare the RF classification results with the correct classification; compute the false classification number ( $P$ ) by RF; and then, the OOB error is $P / S$. It has been proven that it is an unbiased estimation; therefore, in RF, it is not necessary to apply cross validation to get the unbiased estimation for a test set error.

\subsection{Measurement}

The microstrip patch antenna, which is insensitive to the human body, is used for on-body channel measurement at $2.45 \mathrm{GHz}$. The antenna is fabricated on an RT/Duroid board of dielectric constant $\varepsilon_{r}=3$ and thickness of $1.524 \mathrm{~mm}$. The board size is $60 * 80 \mathrm{~mm}^{2}$ and the patch size is $34.95 * 39.5 \mathrm{~mm}^{2}$. A HP8720ES vector network analyzer was used to obtain the $S_{21}$ between two patch antennas. Two identical antennas were used and the distance from the human body is $4 \mathrm{~mm}$. At specified frequency, $S_{21}$ magnitude is measured every second. Each posture or scenario lasts for 20 seconds. The positions of the receiving antenna are listed in Table 2(a) and the different scenarios are given in Table 2(b).

\subsection{Prediction results and analysis}

The confusion matrices for the two environments are listed in Table 1. The classification errors are very small except for marginal cases. This is due to the fact that there is a crisscross between two different postures.

Figure 3 showed that the total error is affected by the number of trees in the random forest. It is known that when the number of trees in the forest is not too large $\$ 200)$, the prediction error, both for anechoic chamber and indoor environment, would experience fluctuations; then, with the increase in the number of trees, the error will flatten out. Note that for the anechoic chamber, the fluctuation is more aggressive and the average error is smaller. The reason is that the multipath scattering in the ordinary laboratory will increase the prediction error and weaken the effect from the number of trees. This important finding indicates that the posture detection approach presented in the paper is more appropriate for weak scattering environment. 
Table 2(a) On-body antenna positions

\begin{tabular}{l|l|l|l|l|l}
\hline \hline $\mathrm{Rx} 1$ & $\mathrm{Rx} 2$ & $\mathrm{Rx} 3$ & $\mathrm{Rx} 4$ & $\mathrm{Rx} 5$ & $\mathrm{Rx} 6$ \\
\hline Right chest & Left chest & Left ear & Right ear & Left wrist & Right wrist \\
\hline \hline
\end{tabular}

Table 2(b) Human body postures and corresponding symbols

\begin{tabular}{l|l}
\hline \hline Symbol & Posture \\
\hline A & Still (Standing) \\
\hline B & Body turn left (Standing) \\
\hline C & Body turn right (Standing) \\
\hline D & Body lean forward (Standing) \\
\hline E & Head lean forward (Standing) \\
\hline F & Head turn left (Standing) \\
\hline G & Head turn right (Standing) \\
\hline H & Arms stretch to the sides (Standing) \\
\hline I & Arms above the head (Standing) \\
\hline J & Arms stretch forward (Standing) \\
\hline L & Arms stretch forward (Lower, Standing) \\
\hline L & Move arms, head and body (Standing) \\
\hline M & Arms hang along the body (Sitting) \\
\hline $\mathrm{N}$ & Hands in lap (Sitting) \\
\hline $\mathrm{O}$ & Move arms, head and body (Sitting) \\
\hline P & Still (Standingupright) \\
\hline $\mathrm{Q}$ & Walking \\
\hline R & Move arms, head and body (Walking) \\
\hline \hline
\end{tabular}

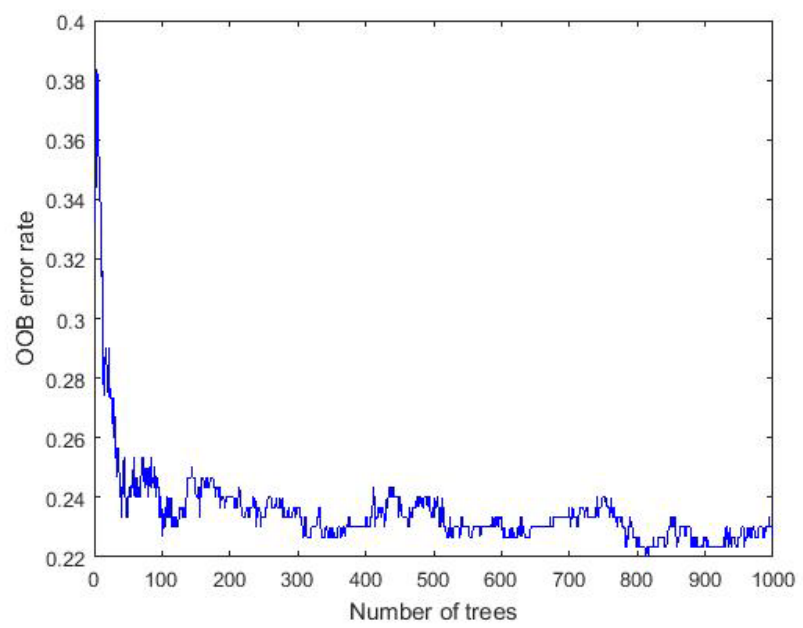

Figure 3(a) Change of errors with the increase of number of trees (anechoic chamber) 


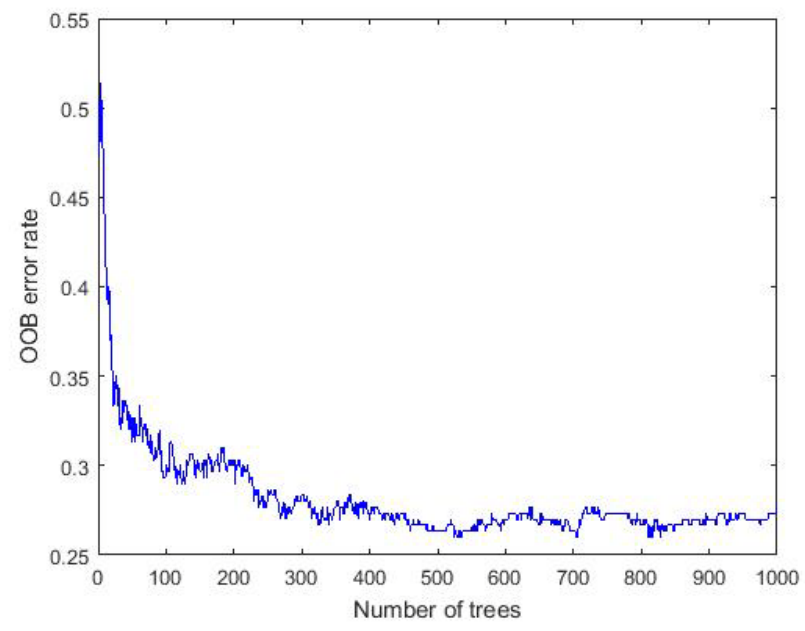

Figure 3(b) Change of errors with the increase of number of trees (indoor environment)

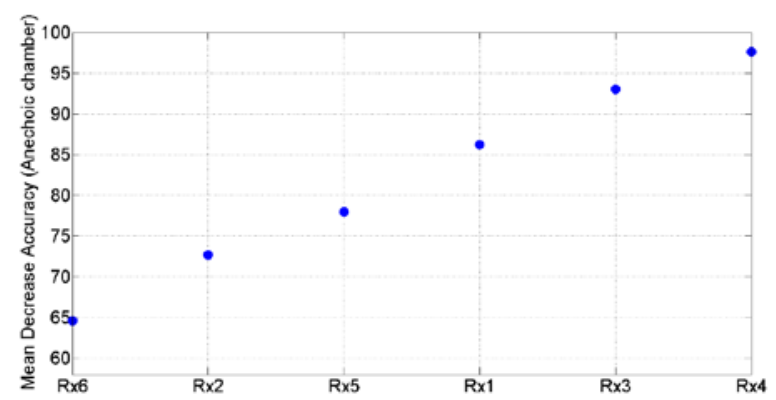

(a) Anechoic chamber

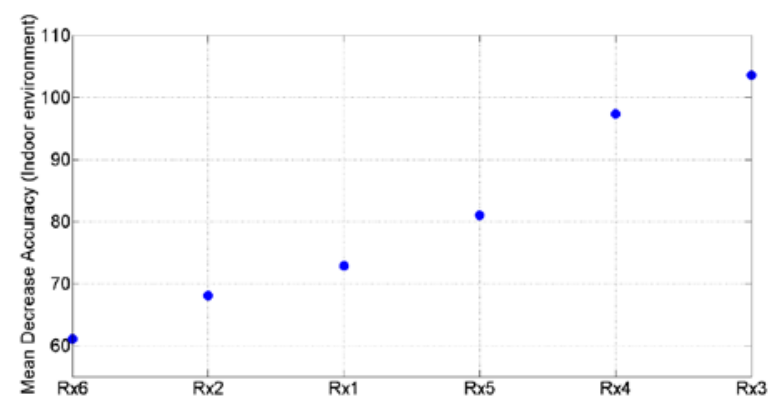

(b) Indoor environment.

Figure 4 The rank of on-body position importance

It can be anticipated that different receiving positions will play various roles in predicting human body postures; therefore, it is necessary and interesting to investigate the rank of importance of different onbody positions. In Figure 4, six on-body receiving positions were ranked in order of importance. In this work, "Mean Decrease Accuracy" was selected as the index to evaluate the importance of the variable (onbody position) [28]. "Mean Decrease Accuracy” can assess the reduction of RF prediction accuracy when a variable is taken as random number. A higher "Mean Decrease Accuracy” means more importance. Comparing horizontal axis in Figure 4 with Table 2(a), it is important to note that the receiving positions 
near the head are the most influential to posture detection, both for anechoic chamber and indoor environment. Slight changes of the order of importance can be observed for these two different propagation environments; however, the main sequences are the same.

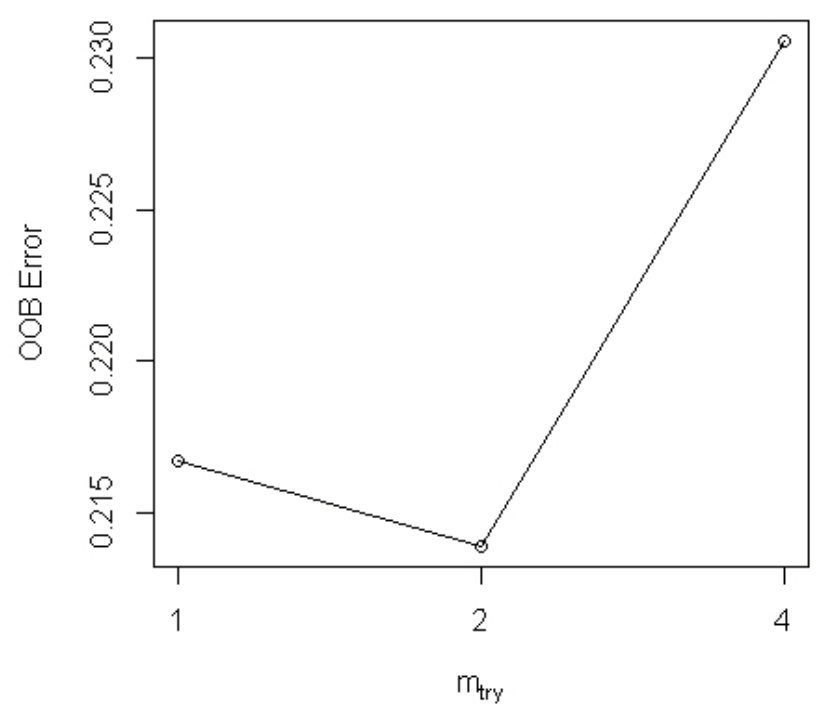

(a) Anechoic chamber

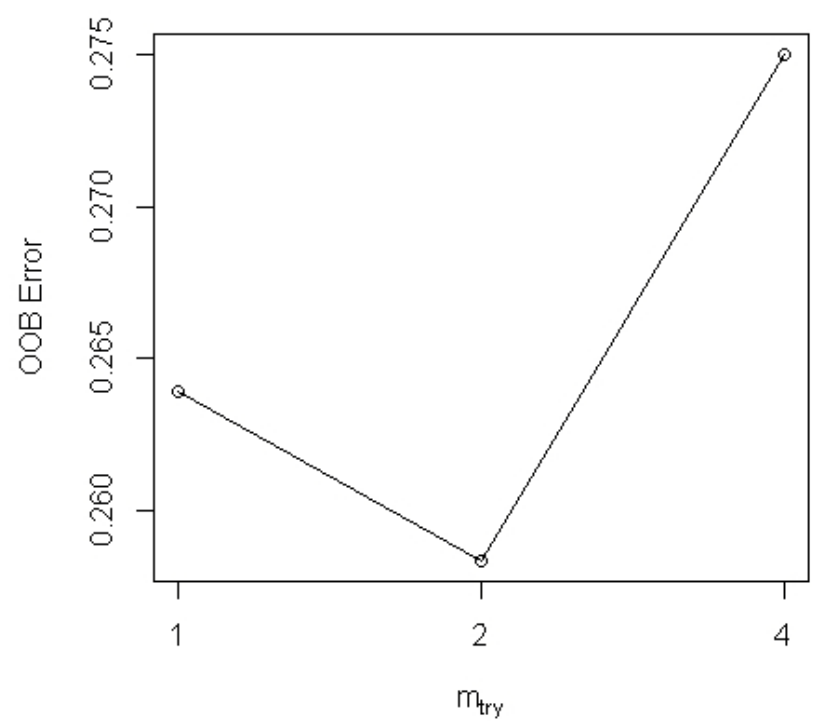

(b) Indoor environment

Figure 5 Mtry parameters in RF and their influence on the OOB errors, the number of trees $=1000$ 
The initial value for mtry parameter is 2, we search the optimal value for the parameter, and the number of trees in the random forest is set to 1000. It is found that the general trends for the two propagation environment are the same; however, in the anechoic chamber, since there is less effects from surroundings, the OOB errors are less, indicating higher prediction accuracy.

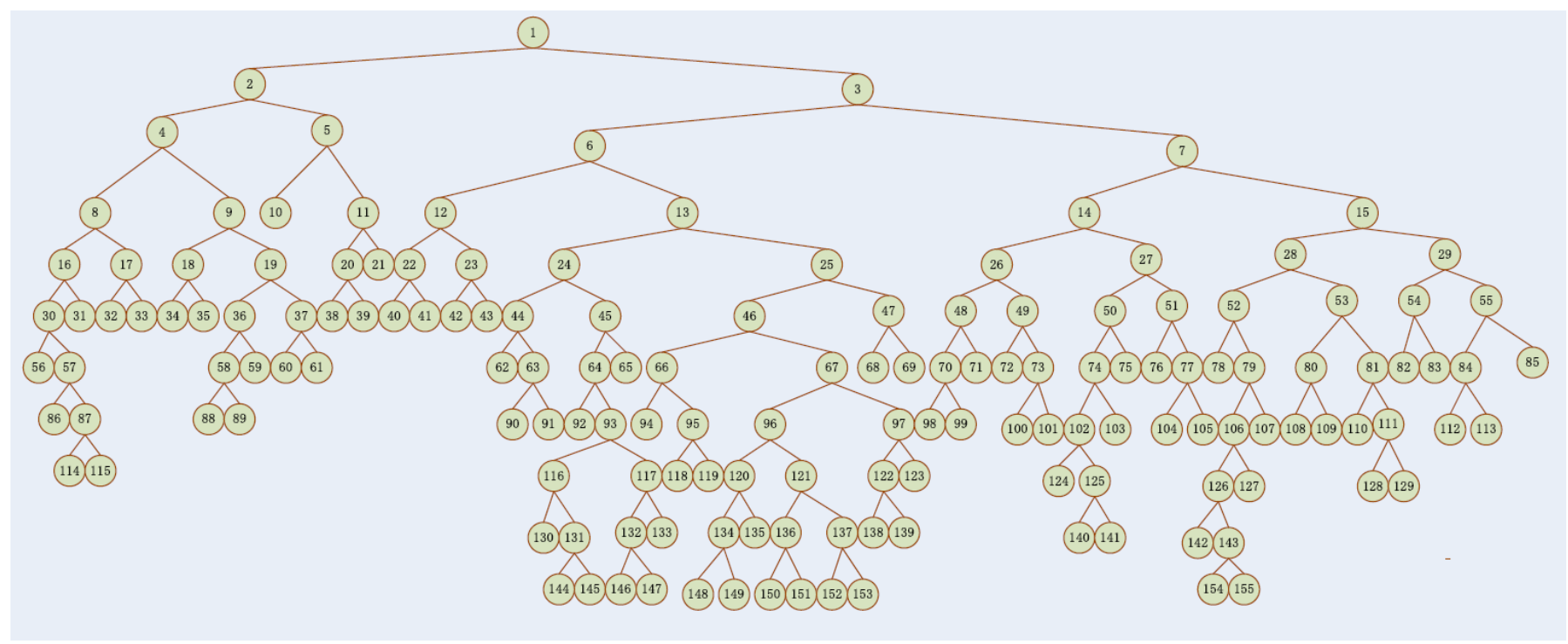

(a) Anechoic chamber

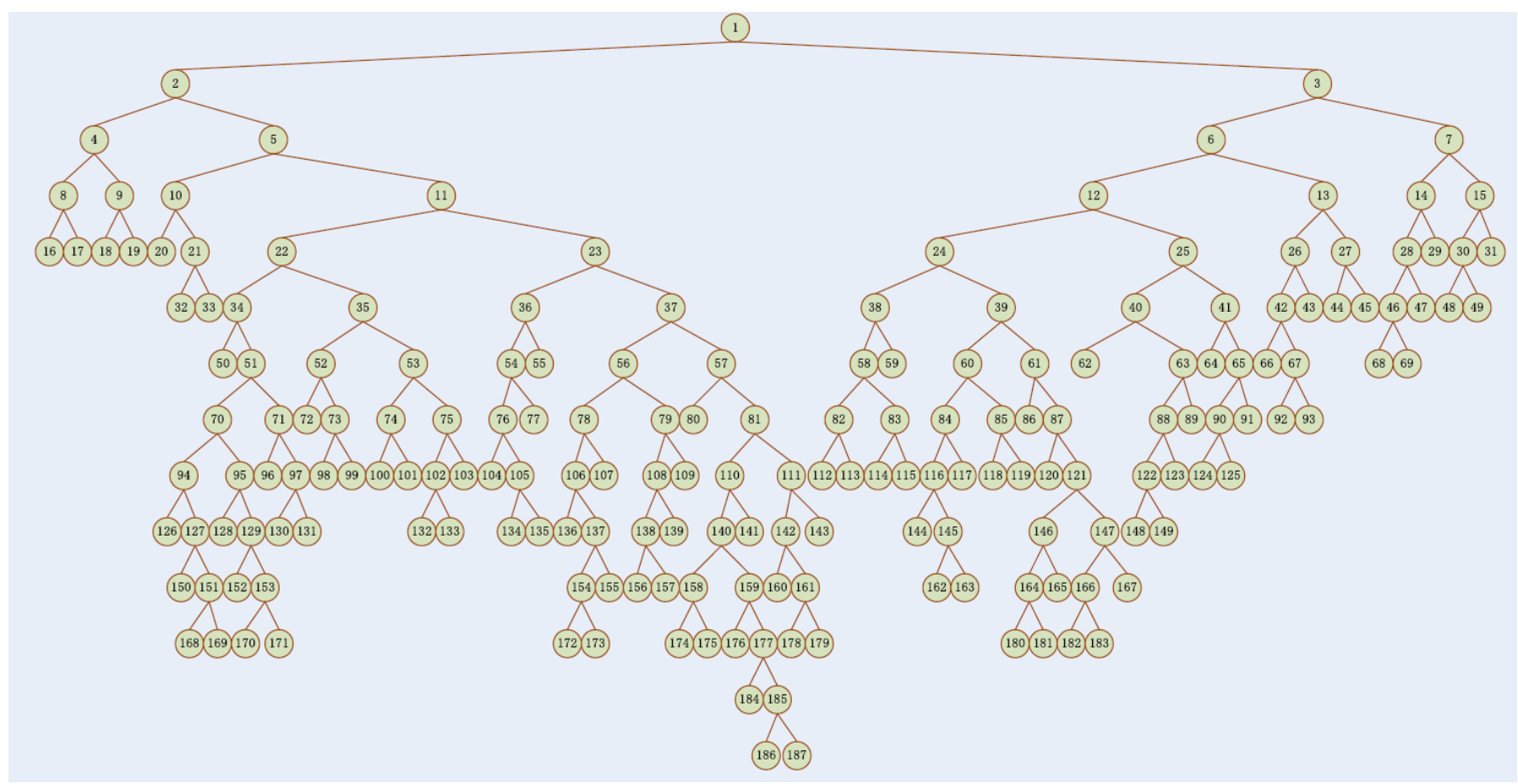

(b) Indoor environment

Figure 6 Specific structures for the 1000th trees considering different environment 


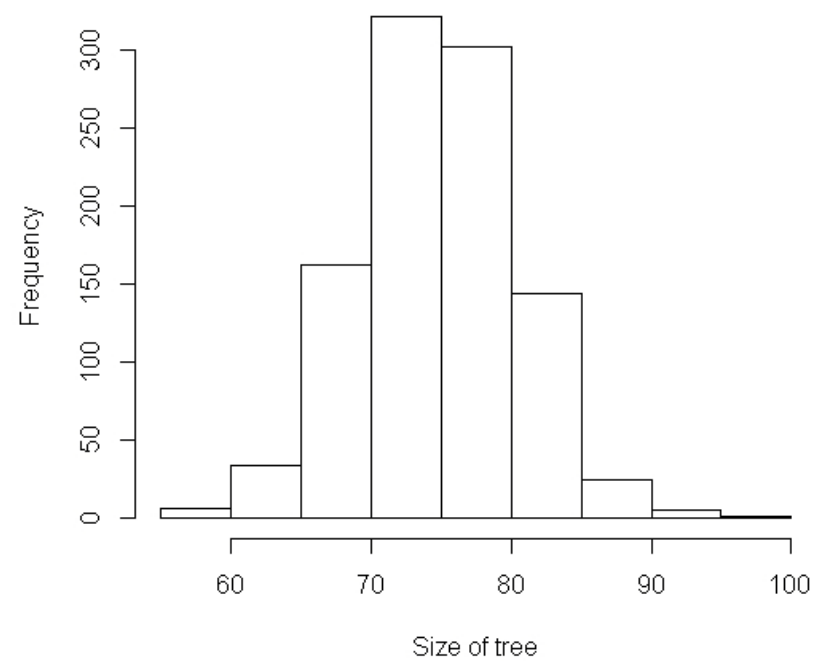

(a) Anechoic chamber.

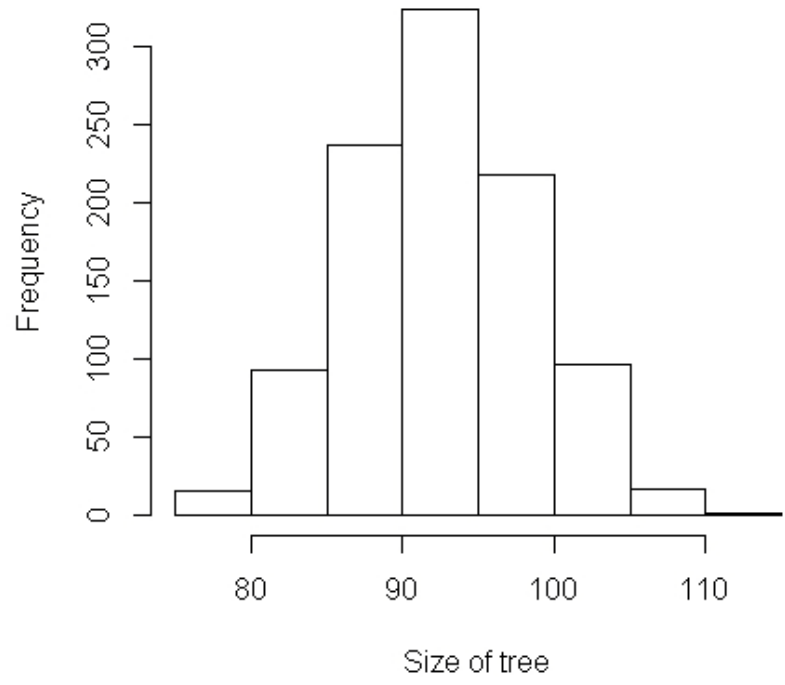

(b) Indoor environment

Figure 7 Size for trees in the random forest considering different environment

The sizes of trees in the random forest are given in figure 7 . In the calculation process, only terminal nodes are considered.

\section{Conclusion}

Posture detection is very significant in assisted living, which is an important component of a smart home. In previous WBANs studies, to the best of authors' knowledge, channel models are determined for specific body postures. Although some non-parametric models have been proposed to consider different 
propagation scenarios, it is still very difficult to extract body posture information from available on-body radio channel characteristics. Under these circumstances, this paper presents a novel approach to detect human body postures from on-body radio propagation information. The framework of the method is based on the combination of decision trees. It is found that the precision of the recognition is perfectly acceptable for the two typical propagation environments. Therefore, in most cases, a subject's postures can be timely detected from on-body sensor nodes.

\section{Acknowledgments}

The work was supported in part by the National Natural Science Foundation of China (Grant No. 61301175), in part by the Fundamental Research Funds the Central Universities, in part by the Project Funded by China Postdoctoral Science Foundation.

\section{References}

[1] AkramAlomainy, “Antennas and Radio Propagation for Body-centric Wireless Networks,” PhD Thesis, May 2007.

[2] ParisaRashidi, Alex Mihailidis, “A Survey on Ambient-Assisted Living Toolsfor Older Adults,” IEEE Journal of Biomedical and Health Informatics, Vol. 17, No. 3, pp. 579-590, May, 2013.

[3] NooritawatiMd Tahir, Aini Hussain, Salina Abdul Samad, Hafizah Husain, MohdYusof Jamaluddin, "SHOCK POSTURE FOR SHAPE MATCHING,” 2nd Information and Communication Technologies, pp. 169-172, 2006.

[4] HomaForoughi, BaharakShakeriAski, HamidrezaPourreza, "Intelligent Video Surveillance for Monitoring Fall Detection ofElderly in Home Environments,” Proceedings of 11th International Conference on Computer and Information Technology, pp. 219-224, 2008.

[5] R. Akmeliawati, F. Dadgostar, S. Demidenko, N. Gamage, Y.C. Kuang, C. Messom, M. Ooi, A. Sarrafzadeh, G. SenGupta, “Towards Real-Time Sign Language Analysisvia Markerless Gesture Tracking,” International Instrumentation and MeasurementTechnology Conference, pp. 1200-1204, 2009.

[6] RohitVerma, Ankit Dev, "Vision based Hand Gesture Recognition Using Finite State Machines and Fuzzy Logic,” International Conference on Ultra-Modern Telecommunications \& Workshops, pp. 1-6, 2009.

[7] S. Bilal, RiniAkmeliawati, MomohJimoh El Salami, Amir A. Shafie, El Mehdi Bouhabba, "A Hybrid Method Using Haar-like and Skin-Color Algorithm for Hand Posture Detection, Recognition and Tracking,” Proceedings of the 2010 IEEE International Conference on Mechatronics and Automation, pp. 934-939, August, Xi'an, China, 2010. [8] Mohammed Anbar, Ahmed manasrah, "An Investigation Towards Worms Detection Approaches Over Network,” 2011 UKSim 13th International Conference on Modelling and Simulation, pp. 266-269, 2011.

[9] Sara Bilal, RiniAkmeliawati, MomohJimoh El Salami, Amir A. Shafie, "Vision-based Hand Posture Detection and Recognition for Sign Language-A study,” 2011 4th International Conference on Mechatronics, pp. 1-6, 17-19, May, Kuala Lumpur, Malaysia. 
[10] Octavian M.S. Dogărescu, Sever S.G. Paşca, “Abnormal Posture Detection Device with Audible Feedback," 2011 7th advanced topics in electrical engineering, pp. 1-4, May, 12-14, 2011.

[11] HristijanGjoreski, MitjaLuštrek, Matjaž Gams, “Accelerometer Placement for Posture Recognition and Fall Detection,” 2011 Seventh International Conference on Intelligent Environments, pp. 47-54, 2011.

[12] Humberto SoutoJ'unior, SoraiaRauppMusse, “Automatic Detection of 2D Human Postures Based on Single Images,” 2011 24th SIBGRAPI Conference on Graphics, Patterns and Images, pp. 48-55, 2011.

[13] Vanco, M., Minarik, I., Rozinaj, G., “Gesture Identification for System Navigation in 3D Scene,” 2012 proceedings ELMAR, pp. 45-48, 2012.

[14] Henry Braun, Humberto SoutoJ'unior, J'ulio C. S. Jacques J'unior, Leandro L. Dihl, Adriana Braun, SoraiaRauppMusse, “Making Them Alive,” 2011 Brazilian games and digital entertainment, pp. 182-188, 2011.

[15] WuttichaiPutchana, SorawatChivapreecha, TulayaLimpiti, "Wireless Intelligent Fall Detection and Movement Classification using Fuzzy Logic,” The 2012 Biomedical Engineering International Conference, pp. 1-5, 2012.

[16] Diana Rojas Sanchez, Lorena Velasquez, Luz Helena Camargo, "Design of an EMG wireless surface EMG 6 channels,” 2013 ISSNIP biosignals and biorobotics, pp. 1-6, 2013.

[17] Alvaro Uribe-Quevedo, Byron Perez-Gutierrez, Cesar Guerrero-Rincon, "Seated tracking for correcting computer work postures,” 2013 9th southern biomedical engineering conference, pp. 169-170, 2013.

[18] MadhatAlsoos, Ammar Joukhadar, “Posture Independent Model for Hand Detectionand Tracking,” $20136^{\text {th }}$ international conference on human system interaction, pp. 175-179, 2013.

[19]SomayehShiravandi, Mohammad Rahmati, Fariborzmahmoudi, "Hand gestures recognition using dynamic Bayesian networks,” 2013 3rd joint conference of AI \& robotics and 5throbocup Iran open international symposium, pp. 1-6, 2013.

[20] WaskithoWibisono, Dedy NurArifin, BaskoroAdiPratomo, Tohari Ahmad, Royyana M Ijtihadie, "Falls Detection and Notification System Using Tri-Axial Accelerometer andGyroscope Sensors of A Smartphone,”2013 Conference on Technologies and Applications of Artificial Intelligence, pp. 382-385, 2013.

[21]SutangSawasdee, SureePumrin, "Elderly Care Notification System usingHand Posture Recognition,” 2014 fourth international conference on digital information and communication technology and its applications, pp. 304309, 2014.

[22] Ines Teyeb, OlfaJemai, MouradZaied, Chokri Ben Amar, “A Novel Approach for Drowsy Driver DetectionUsing Head Posture Estimation and EyesRecognition System Based on Wavelet Network," the 5th international conference on information, intelligence, systems and applications, pp. 379-384, 2014.

[23] Mengyuan Li, Paola Savvidou, Bradley Willis, Marjorie Skubic, "Using the Kinect to Detect Potentially Harmful Hand Postures inPianists," $201436^{\text {th }}$ annual international conference of the IEEE engineering in medicine and biology society, pp. 762-765, 2014.

[24] Leo Breiman, “Random Forests,” Machine Learning, Vol. 45, pp. 5-32, 2001. 
[25] Robert Tibshirani, "Bias, variance and prediction error for classification rules,” Technical Report, Department of Preventive Medicine and Biostatistics and Department of Statistics, University of Toronto, Toronto, Canada.

[26] David H. Wolpert and William G. Macready, “An Efficient Method to Estimate Bagging’s Generalization Error,” SFI Working paper, 1996.

[27] Leo Breiman, “Out-of-bag Estimation,” Technical Report, Statistics Department, University of California, Berkeley, CA, 94708.

[28] Liaw A. Package “random Forest.” http: // stat-www. Berkele.Edu./users /breiman / Random Forests, 2012.

\section{Appendices}

According to Leo's definitions [24]: $\left\{\theta_{k}\right\} k=1,2 \ldots$ is the independent and identical distributed random vectors, $x$ is the input and $h\left(x, \theta_{k}\right), k=1,2 \ldots$ is the classifier.

The generalization error is defined as:

$$
\begin{aligned}
& P E^{*}=P_{X, Y}(m g(X, Y)<0) \\
& =P_{X, Y}\left(P_{\theta}(h(X, \theta)=Y)-\max P_{\theta}\left(h_{j \neq Y}(X, \theta)=j\right)<0\right) .
\end{aligned}
$$

The margin function is defined as:

$$
m g(X, Y)=a v_{k} I\left(h_{k}(X)=Y\right)-\max a v_{k} I\left(h_{k}(X)=j\right),
$$

in the random forest [24], the margin function is defined as:

$$
m r(X, Y)=P_{\theta}(h(X, \theta)=Y)-\max _{j \neq Y} P_{\theta}(h(X, \theta)=j) .
$$

The strength for the classifier is:

$$
s=E_{X, Y} m r(X, Y) .
$$

The expectation of $m r(X, Y)$ is:

$$
P E^{*}=P_{X, Y}(m r(X, Y)<0) .
$$

The prototype for the Chebyshev inequality is:

$$
P(|X-E X| \geq \varepsilon) \leq \frac{D X}{\varepsilon^{2}},
$$

where $E X$ is the expectation, $D X$ is the variance, $\varepsilon$ stands for any constant which is greater than 0 .Then we have:

$$
P(|m r(X, Y)-s| \geq s) \leq \frac{\operatorname{var}(m r(X, Y))}{S^{2}},
$$

where $\operatorname{var}(m r(X, Y))$ is the variance of $m r(X, Y)$. Therefore:

$$
P(m r(X, Y) \geq 2 \text { or } m r(X, Y) \leq 0) \leq \frac{\operatorname{var}(m r(X, Y))}{S^{2}},
$$

Then:

$$
P(m r(X, Y) \leq 0) \leq \frac{\operatorname{var}(m r(X, Y))}{S^{2}},
$$

finally, 


$$
P E^{*} \leq \frac{\operatorname{var}(m r(X, Y))}{S^{2}} .
$$

It is worth mentioning that the derivation process from (7) to (10) can be used as the additional remarks of the Chebyshev inequality and they are not mentioned by Leo's original algorithms. We substitute the definition of variance into the previous definition of margin function for random forest, by changing the sequence of expectations and extracting the same part in the formulas, we obtain:

$$
\begin{aligned}
& \operatorname{var}(m r(X, Y)) \\
& =E\left(m r^{2}(X, Y)\right)-S^{2} \\
& =E_{\theta, \theta^{\prime}}\left(\operatorname{co}_{X, Y}\left(r m g(\theta, X, Y), r m g\left(\theta^{\prime}, X, Y\right)\right)\right) . \\
& =E_{\theta, \theta^{\prime}}\left(\rho\left(\theta, \theta^{\prime}\right) \operatorname{sd}(\theta) \operatorname{sd}\left(\theta^{\prime}\right)\right) \\
& =\bar{\rho}\left(E_{\theta}(\operatorname{sd}(\theta))\right)^{2} \\
& \leq \bar{\rho}\left(1-S^{2}\right)
\end{aligned}
$$

In inequality (11), we omit the definition of $\bar{\rho}$ by Leo:

$$
\bar{\rho}=E_{\theta, \theta^{\prime}}\left(\rho\left(\theta, \theta^{\prime}\right) s d(\theta) s d\left(\theta^{\prime}\right)\right) / E_{\theta, \theta^{\prime}}\left(s d(\theta) s d\left(\theta^{\prime}\right)\right) .
$$

Therefore, finally we have:

$$
P E^{*} \leq \frac{\bar{\rho}\left(1-S^{2}\right)}{S^{2}} .
$$

